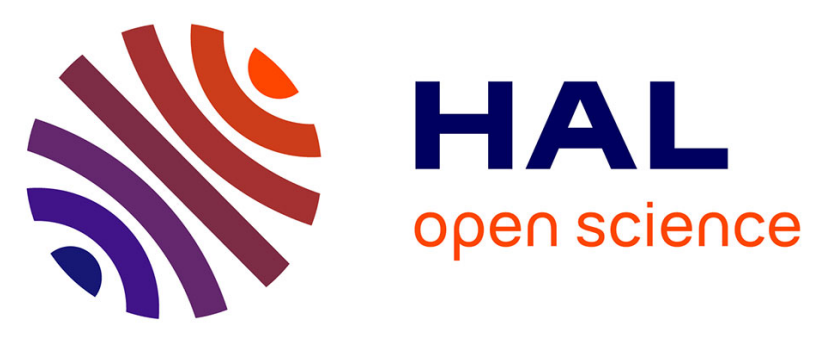

\title{
Pulsed gate bias control of GaN HEMTs to improve pulse-to-pulse stability in radar applications
}

Julien Delprato, Arnaud Délias, Pierre Medrel, Denis Barataud, Michel

Campovecchio, Guillaume Neveux, Audrey Martin, Philippe Bouysse, Jean-Michel Nebus, C Tolant, et al.

\section{To cite this version:}

Julien Delprato, Arnaud Délias, Pierre Medrel, Denis Barataud, Michel Campovecchio, et al.. Pulsed gate bias control of GaN HEMTs to improve pulse-to-pulse stability in radar applications. Electronics Letters, 2015, 51 (13), pp.1023-1025. 10.1049/el.2015.1052 . hal-01286826

\section{HAL Id: hal-01286826 \\ https://hal-unilim.archives-ouvertes.fr/hal-01286826}

Submitted on 11 Mar 2016

HAL is a multi-disciplinary open access archive for the deposit and dissemination of scientific research documents, whether they are published or not. The documents may come from teaching and research institutions in France or abroad, or from public or private research centers.
L'archive ouverte pluridisciplinaire HAL, est destinée au dépôt et à la diffusion de documents scientifiques de niveau recherche, publiés ou non, émanant des établissements d'enseignement et de recherche français ou étrangers, des laboratoires publics ou privés. 


\section{Pulsed gate bias control of GaN HEMTs to improve pulse-to-pulse stability in radar applications}

J. Delprato, A. Delias, P. Medrel, D. Barataud, M. Campovecchio ${ }^{\bowtie}$, G. Neveux, A. Martin, P. Bouysse, J.M. Nebus, C. Tolant and P. Eudeline

A significant improvement is demonstrated in the measured pulse-to-pulse stability of an S-band $6 \mathrm{~W}$ GaN high electron mobility transistor (HEMT) power amplifier by generating an appropriate pulse of the gate bias and thus a warm-up drain current just before each radiofrequency (RF) pulse of a periodic and coherent radar burst. The amplitude and the width of this gate bias pulse preceding each periodic RF pulse of the burst are experimentally varied to investigate the trade-off between the improvement of pulse-to-pulse stability and the total power-added efficiency. Finally, this technique of synchronised warm-up gate bias pulse demonstrated a $10 \mathrm{~dB}$ improvement of measured amplitude pulse-to-pulse stabilities to meet the critical stability requirement below $-55 \mathrm{~dB}$ for the RF power amplifier.

Introduction: Pulse-to-pulse ( $\mathrm{P} 2 \mathrm{P})$ stability is a critical figure of merit of radar transmitters, which quantifies the variations of envelope amplitude and phase over time between consecutive radio-frequency (RF) pulses of a radar burst $[1,2]$. The $\mathrm{P} 2 \mathrm{P}$ stability is a key requirement for the suppression of unwanted radar echoes, called clutter [1]. For GaN high electron mobility transistor (HEMT) power amplifiers (PAs) of radar transmitters, it has already been observed that the P2P stability is mainly affected by low-frequency dispersive effects such as selfheating and traps [1]. Indeed, typical radar sequences may integrate long silence durations between the successive bursts of RF pulses, which lead to exacerbating the thermal effects [3] across the pulsed $\mathrm{RF}$ signals. Therefore, to enhance the P2P stability of a PA, this Letter investigates a pulsed bias control that consists in applying a short gate bias pulse before each RF pulse of the periodic burst to generate a warm-up drain current in the GaN HEMT. The idea is to impose a quasi-steady-state operation of the device during all the RF pulses. In this Letter, the trade-off between P2P stability and efficiency is experimentally characterised for different widths and amplitudes of the warm-up gate bias pulse.

Definition of P2P stability: Typical radar applications make use of irregular RF pulse trains. However, the P2P stability of these complex pulse trains can be analysed through a periodic test signal [1] shown in Fig. $1 a$, which consists of $N$ periodic RF pulses followed by a long silence duration $T_{\mathrm{OFF}}$ (i.e. off-time). Each of the $N$ periodic RF pulses has a pulse width $\mathrm{PW}_{\mathrm{RF}}$ and a pulse-repetition-period $\mathrm{PRP}_{\mathrm{RF}}$, thus defining the on-time $T_{\mathrm{ON}}$ of the periodic RF burst $T_{\mathrm{BURST}}$. The P2P stability is defined at each time $t_{k}$ along $\mathrm{PW}_{\mathrm{RF}}$ by the variations between the envelopes at $t_{k}$ of the $N$ consecutive RF pulses of the burst. The sampling point $t_{k}$ corresponds to the same time position within each RF pulse. In the case of $M$ successive bursts, the root mean square method is used to calculate the amplitude or phase $\mathrm{P} 2 \mathrm{P}$ stability at $t_{k}$, which is defined as

$$
\begin{aligned}
& \overline{\operatorname{Stab}_{\mathrm{RMS}}\left(t_{k}\right)} \\
& \quad=10 \times \log \left(\frac{1}{N-1} \sum_{i=1}^{N-1}\left[\overline{\tilde{x}_{i+1}\left(t_{k}\right)}-\overline{\tilde{x}_{i}\left(t_{k}\right)}\right]^{2}\right)
\end{aligned}
$$

with

$$
\overline{\tilde{x}_{i}\left(t_{k}\right)}=\frac{1}{M} \sum_{m=1}^{M} \tilde{x}_{i m}\left(t_{k}\right) ; \quad 0<t_{k}<\mathrm{PW}_{\mathrm{RF}}
$$

where $\tilde{x}$ stands for the envelope amplitude or phase in the case of amplitude or phase P2P stability, respectively. The indices of $\tilde{x}_{i m}$ refer to the $i$ th pulse of the $m$ th burst. For the sake of clarity, the stability levels reported in this Letter correspond to the worst-case values measured in the first $10 \mu \mathrm{s}$ of RF pulses $\left(0<t_{k}<10 \mu \mathrm{s}\right)$ [1].

Stability measurement setup for pulsed gate bias control: With the aim to mitigate thermal and trapping effects that degrade the P2P stability after long silence duration [1], a gate bias pulse is applied to warm up the GaN HEMT prior to each RF pulse. Fig. $1 a$ shows the ideal (i.e. without instabilities) time-domain envelope of the RF burst with warm-up gate bias pulses. Eight periodic RF pulses $(N=8)$ of $50 \mu \mathrm{s}$ pulse width $\mathrm{PW}_{\mathrm{RF}}$ and $250 \mu$ s pulse period $\mathrm{PRP}_{\mathrm{RF}}$ constitute the RF pulse train with a total duration $T_{\mathrm{ON}}$ of $2 \mathrm{~ms}$. The silence $T_{\mathrm{OFF}}$ between each RF pulse train is $8 \mathrm{~ms}$ long. Fig. $1 b$ shows the ideal envelope of the resulting drain bias current with its warm-up value $I_{\text {warm-up }}$ due to the gate bias pulse and its operation value $I_{\mathrm{DS} 0}$ due to the RF level.
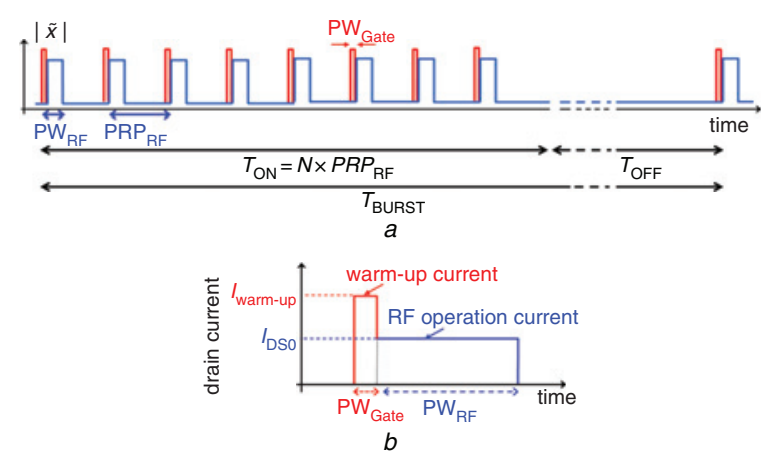

Fig. 1 Ideal time-domain envelopes generated for P2P stability control $a$ Periodic coherent burst made of silence $T_{\mathrm{OFF}}$ that follows $N$ periodic RF pulses $\left(\mathrm{PW} \mathrm{RF}_{\mathrm{RF}}\right.$, each one being preceded by its gate bias pulse $\left(\mathrm{PW}_{\mathrm{Gate}}\right)$

$b$ Ideal envelope of drain bias current during warm-up and RF pulses

The total power-added efficiency $\mathrm{PAE}_{\mathrm{TO}}$ of the PA including the gate bias pulses can be expressed as a function of its initial PAE, as follows:

$$
\begin{aligned}
\mathrm{PAE}_{\mathrm{TOT}} & =\frac{P_{\mathrm{RF}_{\text {out }}}-P_{\mathrm{RF}_{\mathrm{in}}}}{V_{\mathrm{DS} 0} I_{\mathrm{DS} 0}(1+\beta \gamma)}=\frac{\text { PAE }}{(1+\beta \gamma)} \text { with } \gamma=\frac{\mathrm{PW}_{\mathrm{Gate}}}{\mathrm{PW}_{\mathrm{RF}}} ; \\
\beta & =\frac{I_{\text {warm-up }}}{I_{\mathrm{DS} 0}}
\end{aligned}
$$

where $\gamma$ is the pulse-width ratio and $\beta$ is the drain-current ratio between the gate bias pulse ( $\left.\mathrm{PW}_{\mathrm{Gate}}, I_{\mathrm{warm}-u p}\right)$ and the RF pulse ( $\left.\mathrm{PW} \mathrm{RF}_{\mathrm{RF}}, I_{\mathrm{DS} 0}\right)$.

A heterodyne measurement setup of time-domain envelopes was previously developed to characterise the P2P stability of a PA [1] under constant bias conditions. As shown in Fig. 2, the coherent generation and capture of time-domain envelopes for periodic pulsed RF signals are performed by a pulsed RF generator with an internal IQ modulator and an RF heterodyne receiver, respectively. The signal coherence is ensured by a $10 \mathrm{MHz}$ reference clock and the envelope trigger synchronises signal generation and data acquisition. To synthesise the pulsed gate bias proposed in this Letter, the initial setup was modified by connecting an arbitrary waveform generator (AWG) to the gate access of the PA under test. All P2P measurements reported here were performed on a $6 \mathrm{~W}$ GaN HEMT test board (CGH4006P-TB) [4] at an RF frequency of $3 \mathrm{GHz}$.

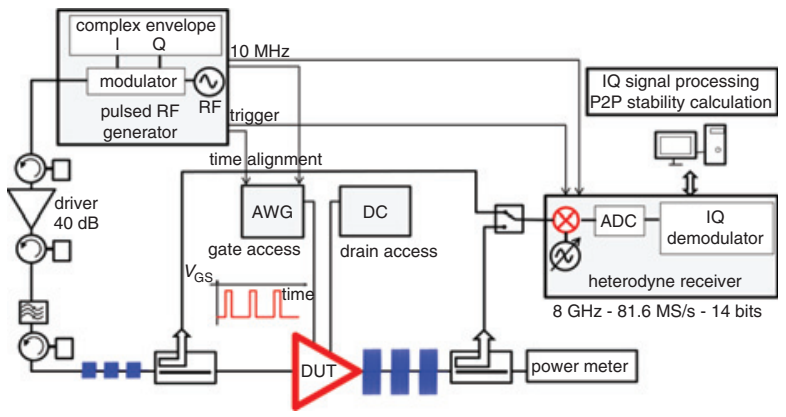

Fig. 2 Heterodyne measurement setup of time-domain envelopes and P2P stability with capability of pulsed gate bias control

P2P stability improvement: First, P2P stabilities were measured under constant bias. Figs. $3 a$ and $b$ show that the reference levels (dashed lines) of amplitude/phase P2P stabilities were measured at -47 and $-62.8 \mathrm{~dB}$, respectively. Then, to improve the amplitude stability below the required level of $-55 \mathrm{~dB}$, the pulsed gate bias control was implemented with a gate voltage varying in amplitude and pulse width. Figs. $3 a$ and $b$ plot the measured stabilities against $\gamma$ and $\beta$ ratios. 

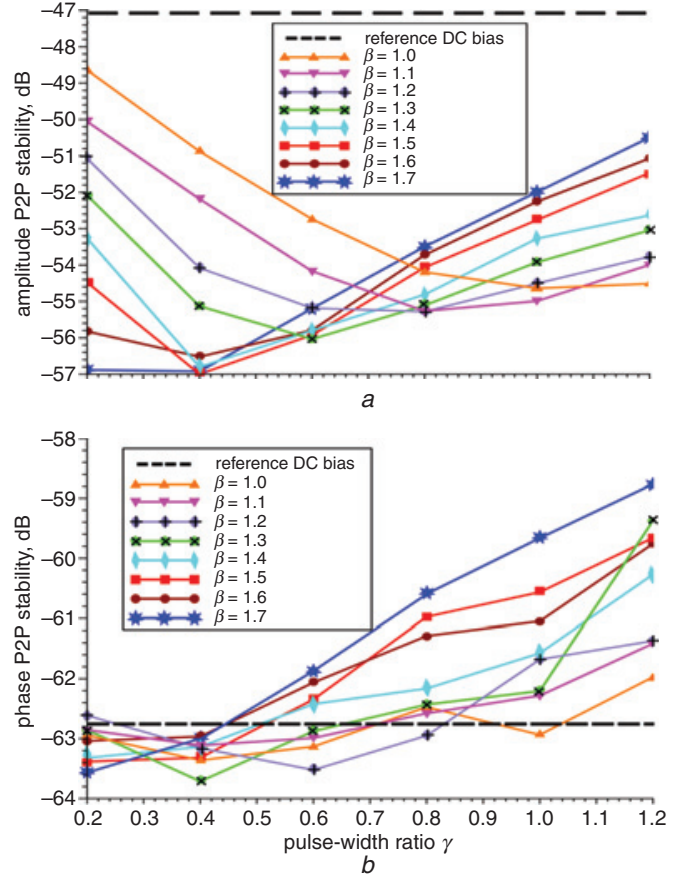

Fig. 3 Measured P2P stabilities and PAE with and without gate bias pulse against pulse-width ratio $\gamma$ and drain-current ratio $\beta$

a Amplitude P2P stability

$b$ Phase P2P stability

The value of $\mathrm{PW}_{\mathrm{Gate}}$ was limited to $10 \mu \mathrm{s}(\gamma=0.2)$ because of the AWG capability while the minimum value of $I_{\text {warm-up }}$ was set to $I_{\text {DSo }}$ $(\beta=1)$ below which the stability requirement of $-55 \mathrm{~dB}$ was never achieved.

Figs. $3 a$ and $b$ show that the gate bias pulse enables achievement of the required level of amplitude P2P stability below $-55 \mathrm{~dB}$ for different combinations of $\gamma$ and $\beta$ ratios. However, due to the impact of gate bias pulses on the total PAE, the best combination of $\mathrm{PW}_{\mathrm{Gate}}$ and $I_{\mathrm{warm} \text {-up }}$ that meets the critical P2P stability requirement while also limiting the decrease of PAE corresponds to $\gamma$ and $\beta$ ratios of 0.2 and 1.7, respectively. The resulting stability performances are given in Table 1 .

Table 1: Comparison of measured P2P stabilities and PAE between reference bias and optimum gate bias pulse to meet stability requirement

\begin{tabular}{|c|c|c|c|}
\hline Gate bias & Amplitude stability $(\mathrm{dB})$ & Phase stability $(\mathrm{dB})$ & PAE (\%) \\
\hline Reference DC bias & -47 & -62.8 & 42 \\
\hline $\begin{array}{c}\text { Gate bias pulse } \\
(\gamma=0.2, \beta=1.7)\end{array}$ & -57 & -63.4 & 32 \\
\hline
\end{tabular}

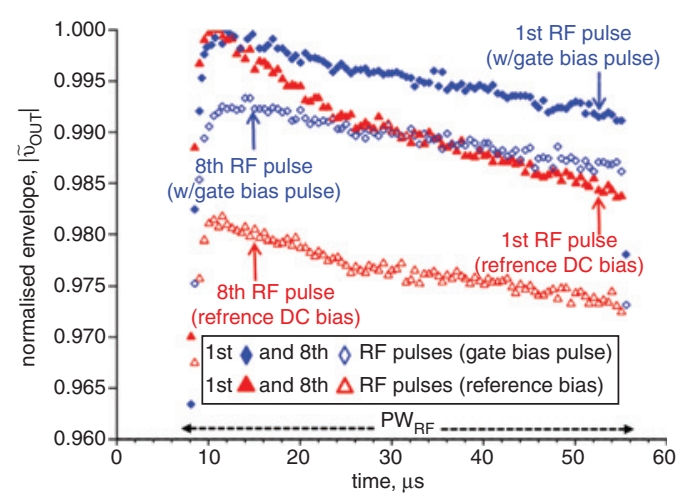

Fig. 4 Normalised envelope amplitudes of first and eighth $R F$ pulses along $P W_{R F}$ that were measured with and without gate bias pulses
To illustrate the relative impact of gate bias pulses along the RF burst, the measured time-domain envelopes of the first and eighth RF pulses with or without gate bias control are superimposed in Fig. 4.

First, it can be observed that the gate bias pulses limit the envelope variation along $\mathrm{PW}_{\mathrm{RF}}$ for both $\mathrm{RF}$ pulses, but much more for the first RF pulse. This leads to a more constant level of amplitude $\mathrm{P} 2 \mathrm{P}$ stability along $\mathrm{PW}_{\mathrm{RF}}$, but the maximum value of stability levels along $\mathrm{PW}$ RF remains the main issue to meet the $\mathrm{P} 2 \mathrm{P}$ stability requirement.

However, the level of P2P stability at $t_{k}(1)$ is directly linked to the envelope variation between RF pulses at the same time $t_{k}$ of $\mathrm{PW}_{\mathrm{RF}}$. In this regard, Fig. 4 shows that the most positive impact of the gate bias pulse on P2P stability levels is observed for the eighth RF pulse. Indeed, in the first $10 \mu \mathrm{s}$ of $\mathrm{PW}_{\mathrm{RF}}$ which correspond to the worst case of stability levels [1], the comparison between envelope amplitudes of the first RF pulse (with or without gate bias pulse) and the eighth RF pulse with the gate bias pulse shows that the stability level would have been equally improved without the gate bias pulse preceding the first RF pulses, and thus without its consumption. Accordingly, an adaptive control using non-systematic gate bias pulses can be implemented for actual radar signals to meet the required stability level while limiting the impact on PAE predicted by (3) in the worst case of systematic gate bias pulses.

Conclusion: This Letter investigates the impact of a gate bias pulse which is applied before each RF pulse of a periodic radar burst to improve its P2P stability degraded by a long silence. All measurements were performed on a $6 \mathrm{~W}$ GaN HEMT test board at an RF carrier frequency of $3 \mathrm{GHz}$ for a worst-case radar burst of $10 \mathrm{~ms}$ integrating a silence of $8 \mathrm{~ms}$. This control technique of systematic gate bias pulses demonstrated a $10 \mathrm{~dB}$ improvement of amplitude P2P stabilities for this worst-case radar burst to meet the critical stability requirement below $-55 \mathrm{~dB}$ without any degradation of phase stability. Therefore, depending on the required level of P2P stability, and on the timedomain characteristics of the radar signal, these results open the way to adaptive bias control techniques of an RF PA for improving their P2P stabilities.

(C) The Institution of Engineering and Technology 2015

Submitted: 27 March 2015 E-first: 10 June 2015

doi: 10.1049/el.2015.1052

One or more of the Figures in this Letter are available in colour online.

J. Delprato, A. Delias, P. Medrel, D. Barataud, M. Campovecchio, G. Neveux, A. Martin, P. Bouysse and J.M. Nebus (XLIM, Université de Limoges/CNRS, 123 Ave. A. Thomas, Limoges 87060, France)

凶 E-mail: michel.campovecchio@xlim.fr

C. Tolant and P. Eudeline (Thalès Air Systems, Ymare 76520, France)

\section{References}

1 Delprato, J., Barataud, D., Campovecchio, M., Neveux, G., Tolant, C., and Eudeline, P.: 'Measured and simulated impact of irregular radar pulse trains on the pulse-to-pulse stability of microwave power amplifiers', IEEE Trans. Microw. Theory Tech., 2014, 62, (12), pp. 3538-3548, doi: 10.1109/78.790650

2 Cicolani, M., Gentile, A., Maccaroni, S., and Marescialli, L.: 'Pulse-to-pulse stability characteristics of robust design centered high performances/low cost T/R module'. European Microwave Conf., Manchester, England, September 2006, pp. 1802-1805, doi: 10.1109/ EUMC.2006.281021

3 King, J.B., and Brazil, T.J.: 'A comprehensive electrothermal GaN HEMT model including nonlinear thermal effects'. IEEE MTT-S Int. Microwave Symp. Dig., Montreal, QC, Canada, June 2012, pp. 1-3, doi: 10.1109/MWSYM.2012.6259423

4 Cree Inc.: 'CGH40006P: 6 W, RF Power GaN HEMT', http://www.cree. com/ /media/Files/Cree/RF/Data\%20Sheets/CGH40006P.pdf, accessed March 2015 